\title{
The Validation of the Utrecht Work Engagement Scale for Emergency Medical Technicians in Gauteng ${ }^{1}$
}

\author{
J L P Naudé and S Rothmann
}

WorkWell: Research Unit for People, Policy and Performance, North-West University

\begin{abstract}
The objectives of this study were to validate the Utrecht Work Engagement Scale (UWES) for emergency medical technicians in the Gauteng Province of South Africa and to determine its construct equivalence and bias for different language groups. A cross-sectional survey design was used with a convenient sample $(N=318)$ of emergency medical technicians in Gauteng. The UWES and a biographical questionnaire were administered. A two-factor model of work engagement, consisting of Vigour/Dedication and Absorption was found. Exploratory factor analysis with target rotations confirmed the construct equivalence of the work engagement construct for white and black employees.
\end{abstract}

JEL M54

\section{1 Introduction}

Researchers and practitioners in psychology are increasingly questioning the prevailing postSecond World War paradigm, described as the pathogenic paradigm where the orientation towards the abnormal, the origin of the "pathos" (disease, ill-health) is taken as the starting point of interventions and strategies aimed at fixing and treating ill-health (Seligman, 2002). This paradigm assumes maladjustment and ill-health of individuals and neglects positive aspects of human functioning (Barnard, 1994). The prevalence of the pathogenic paradigm in the health and social sciences is confirmed by Diener, Suh, Lucas and Smith (1999) reporting that 17 times more scientific articles were published on negative feelings than on positive feelings.

Recently, the field of psychology has been subjected to a transformation, in essence questioning many strongly held beliefs and premises at an individual, group and metatheoretical level (Snyder \& Lopez, 2002). The emergence of a new thinking-set or paradigm takes into account individual strengths and resources, enabling the studying of "normal" or superhuman functioning which could previously not be understood in a problemfocused framework (Strümpfer, 2001). Seligman and Csikszentmihalyi (2000) call this the rising of "positive psychology", a move from a preoccupation with the worst things in life towards also building and investigating positive qualities. According to these authors, psychology is not just the study of pathology, weakness and damage, but also the study of strength and virtue.

Similar tendencies can be detected in the burnout research literature. Empirical studies revealed that some employees, regardless of high job demands and long working hours, do not develop burnout in comparison to others but seemed to find pleasure in hard work and dealing with job demands (Schaufeli \& Bakker, 2001). Consequently, theoretical and empirical studies commenced on the concept of engagement, theoretically viewed as an antithesis of the burnout construct.

Development of the engagement construct took two different, but related paths. Firstly, Maslach and Leiter (1997) rephrased burnout as an "erosion of engagement with the job". 
Subjective experience of work that started out as important, meaningful and challenging becomes unpleasant, unfulfilling and meaningless. Work engagement, according to these authors, is characterised by energy, involvement and efficacy, the direct opposites of burnout, namely exhaustion, cynicism and lack of professional efficacy respectively. Consequently, work engagement could theoretically be measured by means of the Maslach Burnout Inventory (MBI) when low scores on exhaustion and cynicism, and high scores on professional efficacy are obtained.

The second path was taken by Schaufeli and his colleagues, agreeing in part with the description of engagement proposed by Maslach and Leiter (1997), that engagement be measured with a different instrument worthy of operationalisation in its own right (Schaufeli, Salanova et al., 2002). They further argue that the simultaneous empirical investigation of burnout and engagement would be impossible with one instrument. Based on a theoretical analysis, burnout and engagement were conceptually related to each other, resulting in two work-related dimensions of well-being being identified, namely (1) activation, ranging from exhaustion to vigour, and (2) identification, ranging from cynicism to dedication (Schaufeli \& Bakker, 2001). Also, personal accomplishment and absorption were included in the burnout and engagement constructs respectively, but not in an antithetical manner. It was argued that personal accomplishment was added only afterwards in the development of the Maslach Burnout Inventory (MBI) when a third factor was discovered during a factoranalysis of a preliminary version of the MBI (Maslach, 1993). Similarly, absorption was discovered as a related dimension of the work engagement construct during 30 in-depth interviews (Schaufeli et al., 2001).

Work engagement is defined as a positive, fulfilling, work-related state of mind that is characterised by vigour, dedication and absorption. Furthermore, it is not a momentary and specific state, but a more persistent and pervasive affective-cognitive state which is not focussed on a particular object, event, individual or behaviour (Schaufeli, Salanova et al., 2002).
Vigour is characterised by high levels of energy and mental resilience while working, the willingness to invest effort in one's work, not being easily fatigued and the ability to persist even in the face of difficulties. Dedication is characterised by a sense of significance in one's work, feeling enthusiastic, inspired, proud and by viewing it as a challenge. Absorption is characterised by being totally happily immersed in one's work to the extent that it is difficult to detach oneself from it.

Work engagement, therefore, can be distinguished but not divorced from burnout in terms of its structure and operationalisation. Work engagement is theoretically viewed as the opposite end of the continuum from burnout, which cannot be effectively measured by the Maslach Burnout Inventory (MBI) but with its own survey, the Utrecht Work Engagement Scale (UWES) (Schaufeli Salanova, GonzálesRomá \& Bakker, 2002).

Since the development of the UWES by Schaufeli, Salanova, et al. (2002), only a couple of studies could be found in the literature regarding work engagement. Schaufeli, Martinez et al. (2002) conducted a confirmatory factor-analytical cross-national study amongst students from three different countries. Only one study in this regard could be found in South Africa, namely the study of Storm and Rothmann (2003) in the South African Police Service. As a result, information resources regarding the internal consistency, construct validity and comparability across cultural groups for the UWES, are lacking, especially in the multicultural South African context.

Not only is it important to obtain a valid and reliable measurement of engagement in South Africa from an empirical point of view, but also to enable the individual measurement of engagement in a valid and reliable manner in the emergency worker context in South Africa. In line with recommendations of Poortinga (1989) and Van de Vijver and Leung (1997) measurement equivalence and bias should be tested for in contexts where differences in scores could be attributed to cultural influences in terms of item meaning and understanding, rather than differences resulting from the measuring of the constructs by the measuring 
instruments. If cultural influences are not accounted for, invalid conclusions regarding the constructs under study could be made. Where measurement equivalence is concerned with measurement and the comparability of scores, bias is concerned with factors that influence the validity of cross-cultural comparisons.

According to Van de Vijver and Leung (1997), construct equivalence (also known as structural equivalence), indicates the extent to which the same construct is measured across the cultural groups under study-in other words the comparison of cultural groups, because their scores are related to the same construct. On the other hand, in the case of structural inequivalence, no comparison can be made because scores obtained are not related to the same construct.

Item bias, the second important computation in cross-cultural settings, concerns aspects of measurement validity in inter-cultural group comparisons (Van de Vijver \& Leung, 1997). An unbiased item would provide the same average score on an item if two people from different cultural groups are similar in terms of the construct measured by this item. Stated differently, individuals with an equal standing in terms of the underlying construct measured by the instrument would obtain the same score on a given item, irrespective of group membership. Bias can be caused by incidental differences in appropriateness of item content, inadequate item formulation and translation, but also from response characteristics of the sample and administration effects. The danger associated with bias is that it would lower the equivalence of the measuring instrument. Two types of bias can be distinguished, namely uniform and nonuniform bias (Van de Vijver \& Leung, 1997). Uniform bias refers to the main effects of cultural differences, in other words the influence of bias on an item is consistent for all the score levels of that particular item. Non-uniform bias refers to the interaction effects of cultural differences and score level, indicating that across all score levels of an item significantly larger differences in terms of a particular item exists in one group, when compared to the other group across the different score levels for the specific item (Mellenbergh, 1982).
The objectives of this study were to determine the item bias, construct equivalence, construct validity and internal consistency of the UWES in a sample of emergency health technicians.

\section{2}

\section{The Utrecht Work Engagement Scale}

Schaufeli, Salanova, et al. (2002) developed the Utrecht Work Engagement Scale (UWES) and reported acceptable internal consistency for it. Recent confirmatory factor-analytic studies confirmed the factorial validity of the UWES (Schaufeli, Martinez et al., 2002). The findings showed internal consistent results for the three scales of the UWES. In a sample of undergraduate students $(N=314)$ and a sample of employees $(N=619)$ adequate Cronbach alphas were found as follows: Vigour (6 items), $\alpha=0.68$ and 0.80 ; Dedication (5 items), $\alpha=$ 0.91 for both samples and Absorption (6 items), $\alpha=0.73$ and 0.75 . The scales seem to be moderately to strongly related (mean $r=0.70$ ). Also, the fit of the hypothesised three-factor model with the data was found to be superior to the one-factor solution (Maslach, Schaufeli \& Leiter, 2001; Schaufeli, Martinez et al., 2002).

In a cross-cultural study regarding the UWES for students in Spain, Portugal and the Netherlands, the factorial validity of the UWES was confirmed and the internal consistency of the scales was found to be satisfactory (Schaufeli, Martinez et al., 2002). Factor loadings of Absorption were found to be invariant across all samples, while factor loadings of Vigour were invariant for only two of the three groups. The three-factor model fit to the data was found to be superior in all three samples after removing three items. Internally consistent Cronbach alphas ranged from 0.65 to 0.79 for Vigour (5 items); 0.77 to 0.85 for Dedication (5 items); and 0.65 and 0.73 for Absorption (4 items).

Only one study regarding the internal consistency, factorial validity, structural equivalence and bias could be found in South Africa. In their study, Storm and Rothmann (2003) found that a one-factor model fitted the data the best in a sample of police members in South 
Africa $(N=2396)$. Although a three-factor model was also initially tested and satisfactory results obtained, the fit with the data was superior for a onefactor model. Internal consistencies of the three subscales were determined at 0.78 (Vigour); 0.89 (Dedication) and 0.78 (Absorption). No evidence of structural inequivalence or item bias was found for the UWES.

The research hypotheses pertaining to the present study can be formulated as follows:

H1: The construct equivalence of the Utrecht Work Engagement Scale (UWES) is acceptable and none of the items are biased towards different race groups of emergency medical technicians in Gauteng.

H2: The Utrecht Work Engagement Scale (UWES) is an internally consistent and valid measurement for the work engagement construct for emergency medical technicians in Gauteng.

\section{3}

\section{Method}

\subsection{Research design}

A cross-sectional survey design was used to reach the objectives of this research.

\subsection{Sample}

A convenient sample of emergency medical technicians in the different regions of Gauteng, namely West Rand, Ekurhuleni, Sedibeng, Johannesburg Metropolitan, Tshwane, Kungwini and Nokeng Tsa Taemane was taken. The total population of 2100 emergency medical technicians in Gauteng was targeted. A response rate of 21.6 per cent was achieved due to the nature of the job, e.g. call-outs, rotating working schedules and leave. Descriptive information of the sample is given in Table 1 .

The sample consisted mainly of Afrikaansspeaking, married men (58.4 per cent) with a tertiary education (diploma). The mean age of the participants was 33.13 years while the average length of service was 9.68 years. Only 11.88 per cent of the sample had a qualification lower than Grade 12. A total of 64.8 per cent of the participants were white, while 35.2 per cent were black.
Table 1

Characteristics of the participants

\begin{tabular}{|c|c|c|}
\hline Item & Category & Percentage \\
\hline $\begin{array}{l}\text { Home } \\
\text { language }\end{array}$ & $\begin{array}{l}\text { Afrikaans } \\
\text { English } \\
\text { African }\end{array}$ & $\begin{array}{l}45.00 \\
19.80 \\
35.20\end{array}$ \\
\hline Position & $\begin{array}{l}\text { Management } \\
\text { Medical } \\
\text { specialists } \\
\text { Emergency } \\
\text { medical } \\
\text { technicians } \\
\text { Support services }\end{array}$ & $\begin{array}{c}16.70 \\
5.00 \\
72.00 \\
6.30\end{array}$ \\
\hline Area & $\begin{array}{l}\text { West Rand } \\
\text { Ekurhuleni } \\
\text { Sedibeng } \\
\text { Johannesburg } \\
\text { Metro } \\
\text { Tshwane } \\
\text { Kungwini } \\
\text { Nokeng Tsa } \\
\text { Taemane }\end{array}$ & $\begin{array}{c}11.90 \\
45.90 \\
17.00 \\
9.40 \\
\\
8.20 \\
4.70 \\
2.80\end{array}$ \\
\hline Gender & $\begin{array}{l}\text { Male } \\
\text { Female }\end{array}$ & $\begin{array}{l}77.72 \\
22.28\end{array}$ \\
\hline
\end{tabular}

\subsection{Measuring instruments}

The Utrecht Work Engagement Scale (UWES) (Schaufeli Salanova, González-Romá, \& Bakker, 2002) was used in the present study. Also, biographical information was gathered regarding language, race, position, education and gender.

The Utrecht Work Engagement Scale (UWES) (Schaufeli, Salanova et al., 2002) measures levels of engagement. Initially engagement was viewed as the positive antithesis of burnout, but according to the scale developers, it can be operationalised in its own right. The UWES is scored on a 7-point frequency scale, ranging from 0 (never) to 6 (every day). Three dimensions of engagement can be distinguished, namely Vigour (5 items; e.g. "I am bursting with energy in my work"), Dedication (5 items; e.g. "I find my work full of meaning and purpose") and Absorption (5 items; e.g. "When I am working, I forget everything else around me"). Engaged individuals are characterised by high levels of Vigour and Dedication and also elevated levels of Absorption. In terms of 
internal consistency, reliability coefficients for the three subscales have been determined between 0.68 and 0.91 . Improvement of the alpha coefficient (ranging from 0.78 to 0.89 ) seems possible without adversely affecting the internal consistency of the scale (Storm \& Rothmann, 2003). In light of the fact that most items on the UWES are framed in a positive manner, it was decided to include and mix the MBI-HSS items in one questionnaire. The latter is predominantly phrased in a negative manner and should guard against the possibility of response sets.

\subsection{Statistical analysis}

The statistical analysis was carried out with the SPSS program (SPSS, 2003). In the first step, means, standard deviations, skewness and kurtosis were determined to describe the data. The reliability and validity of the UWES were determined by means of Cronbach alpha coefficients, as well as exploratory factor analyses.

Item level analysis (item bias analysis) was performed by using analysis of variance (ANOVA) for the UWES (yielding intervallevel scores). The assumption is that an item is unbiased if persons from different race groups with an equal standing on the theoretical construct underlying the instrument have the same expected score on the item (e.g. Van de Vijver \& Leung, 1997). Although several statistical techniques are available for analysing item bias, analysis of variance has the advantage of computational simplicity, robustness and the possibility to study both uniform and nonuniform bias (Mellenbergh, 1982). Therefore, analysis of variance was used in the present study. The item score was the dependent variable, while race and score levels were the independent variables. A significant main effect of race group was taken to point to uniform bias and a significant interaction of score level and race group pointed to non-uniform bias.

Construct equivalence of the UWES was also performed. Construct equivalence can be investigated with several techniques, such as factor analysis, cluster analysis, and multidimensional scaling or other dimensionality-reducing techniques (Van de Vijver \& Leung, 1997). The basic idea behind the application of these techniques is to obtain a structure in each culture, which can then be compared across all cultures involved. Factor analysis is the most frequently employed technique for studying construct equivalence. In the current study both exploratory and confirmatory models could have been used. Given that there is information about the composition of the instrument (on the basis of previous studies), the choice for confirmatory factor analysis may seem obvious. However, the current authors used exploratory factor analysis for a pragmatic reason. The UWES is a recently developed measuring instrument, and only one other study regarding its validity in South Africa was found. Also, the authors had negative experiences with the use of confirmatory models in studying the construct validity of the UWES. The main problem in the application of confirmatory models is their fit to the data, which is almost always very bad. It is usually not clear whether the reasons for the poor fit are serious and should lead to a reformulation of the model or are trivial and do not challenge the underlying model.

Exploratory factor analysis was therefore used to examine construct equivalence. A principal components analysis was conducted to determine the number of factors of the UWES in the total sample. Subsequently, a direct oblimin rotation was used to determine the solution for each race group. Factors obtained in each group were compared (after target rotation). The agreement was evaluated by a factor congruence coefficient, Tucker's phi (Van de Vijver \& Leung, 1997). Values above 0.90 are taken to point to essential agreement between cultural groups, while values above 0.95 point to very good agreement. A high agreement implies that the factor loadings of the lower and higher level are equal up to a multiplying constant. (The latter is needed to accommodate possible differences in eigenvalues of factors for the race groups). 


\section{4 \\ Results}

Because of the composition of the sample, it was decided to conduct the analyses in this study on race groups rather than language groups. Although the best strategy would have been to define cultural groups in terms language, the sample sizes of the language groups were not large enough to satisfy the assumptions of the statistical techniques which were employed.

Firstly, bias analysis was conducted to identify items which show uniform or non-uniform bias for the two race groups. Score groups were compiled, based on the total score on the UWES. A total of four score levels was obtained by using percentiles, making it possible to assign at least 50 respondents to each score group. Two effects were tested for significance in the subsequent variance analysis, namely the main effects of culture (uniform bias) and interaction effects of culture and score level (non-uniform bias). If both the main effect of culture and the interaction of culture and score level are found to be non-significant, the item is taken to be unbiased. The results of the individual item analysis of variance for the 15-item UWES are presented in Table 2.

\section{Table 2}

Item bias analyses of the UWES

\begin{tabular}{|l|c|c|}
\hline Item & Uniform Bias & Non-uniform Bias \\
\hline & $p$ & $p$ \\
UWES1 & 0.10 & 0.75 \\
UWES4 & 0.66 & 0.00 \\
UWES8 & 0.03 & 0.78 \\
UWES12 & 0.03 & 0.66 \\
UWES15 & 0.15 & 0.07 \\
UWES2 & 0.00 & 0.00 \\
UWES5 & 0.16 & 0.04 \\
UWES7 & 0.78 & 0.92 \\
UWES10 & 0.09 & 0.76 \\
UWES13 & 0.48 & 0.42 \\
UWES3 & 0.26 & 0.47 \\
UWES6 & 0.23 & 0.78 \\
UWES9 & 0.25 & 0.38 \\
UWES11 & 0.71 & 0.83 \\
UWES14 & 0.87 & 0.39 \\
\hline
\end{tabular}

$* \eta^{2}>0.06-$ Practically significant (medium effect)
According to Table 2, no significant eta square $\left(\eta^{2}\right)$ value was obtained for any item of the UWES.

A simple principal components analysis was conducted on the 15 items of the UWES on the total sample of emergency medical technicians. Analysis of the eigenvalues (larger than 1) and scree plot indicated that two factors could be extracted. Next, principal component analysis with a direct oblimin rotation was used in carrying out factor analyses per race group. The pattern matrices for whites and blacks are reported in Table 3.

\section{Table 3}

Pattern matrix of the 15-item UWES for Whites and Blacks

\begin{tabular}{|c|c|c|c|c|c|}
\hline \multicolumn{3}{|c|}{ WHITE } & \multicolumn{3}{|c|}{ BLACK } \\
\hline Item & $\begin{array}{c}\text { Factor } \\
1\end{array}$ & $\begin{array}{c}\text { Factor } \\
2\end{array}$ & Item & $\begin{array}{c}\text { Factor } \\
1\end{array}$ & $\begin{array}{c}\text { Factor } \\
2\end{array}$ \\
\hline UWES1 & 0.72 & -0.08 & UWES1 & 0.44 & 0.09 \\
\hline UWES2 & 0.72 & 0.03 & UWES2 & 0.77 & -0.11 \\
\hline UWES3 & 0.34 & 0.38 & UWES3 & 0.34 & 0.44 \\
\hline UWES4 & 0.81 & -0.02 & UWES4 & 0.94 & -0.1 \\
\hline WES5 & 0.81 & 0.02 & UWES5 & .81 & 0.0 \\
\hline UWES6 & -0.18 & 0.67 & UWES6 & -0.20 & 0.69 \\
\hline UWES7 & 0.84 & 0.04 & UWES7 & 0.66 & 0.25 \\
\hline UWES8 & 0.73 & 0.02 & UWES8 & 0.79 & -0.09 \\
\hline UWES9 & 0.26 & 0.5 & UWES9 & 0.42 & 0.27 \\
\hline UWES10 & 0.25 & 0.54 & UWES10 & 0.71 & -0.06 \\
\hline UWES11 & 0.10 & 0.67 & UWES11 & 0.26 & 0.54 \\
\hline UWES12 & 0.23 & 0.45 & UWES12 & 0.18 & 0.39 \\
\hline 3 & 0.31 & 2 & UWES13 & 0.47 & 0.42 \\
\hline UWES14 & 0.38 & 0.32 & UWES15 & -0.14 & 0.82 \\
\hline V & .10 & 0.48 & UWES16 & 0.07 & 0.63 \\
\hline
\end{tabular}

The pattern matrices of the two-factor solutions for whites and blacks were then used as input for an exploratory factor analysis with target rotations. The two-factor structure was compared across groups by rotating one solution to the other. After target rotation, the following Tucker's phi coefficients were obtained: a) Factor $1=0.93$, and b) Factor $2=0.82$. 
Although the Tucker's phi coefficient for Factor 1 compared favourably with the guideline of 0.90 , it is clear that Factor 2 showed an unacceptable low equivalence for the two race groups.

Inspection of Table 3 revealed that three items were complex and problematic. These items are: a) Item 9 - "I feel happy when I am engrossed in my work." This item loaded on different factors for whites and blacks. b) Item 10 - "I am proud of the work that I do." This item also loaded on different factors for whites and blacks. c) Item 14 - "I get carried away by my work." For whites, this item loaded on both factors, while it loaded strongly (0.82) on Factor 2 in the black group.

After removal of the three items, a simple factor analyses was conducted again. The scree plot and eigenvalues showed two factors which explained 50.17 per cent of the total variance. The pattern matrices for the white and black groups are reported in Table 4.

\section{Table 4}

Pattern matrix of the 12-item UWES for Whites and Blacks

\begin{tabular}{|lccccc|}
\hline \multicolumn{3}{c}{ WHITE } & \multicolumn{3}{c|}{ BLACK } \\
\hline Item & Factor & Factor & Item & Factor & Factor \\
& $\mathbf{1}$ & $\mathbf{2}$ & & $\mathbf{1}$ & $\mathbf{2}$ \\
\hline UWES1 & $\mathbf{0 . 6 6}$ & -0.00 & UWES1 & $\mathbf{0 . 3 9}$ & 0.15 \\
\hline UWES2 & $\mathbf{0 . 7 6}$ & -0.04 & UWES2 & $\mathbf{0 . 7 9}$ & -0.09 \\
\hline UWES3 & 0.38 & $\mathbf{0 . 3 8}$ & UWES3 & 0.39 & $\mathbf{0 . 3 8}$ \\
\hline UWES4 & $\mathbf{0 . 8 1}$ & -0.02 & UWES4 & $\mathbf{0 . 9 6}$ & -0.14 \\
\hline UWES5 & $\mathbf{0 . 8 4}$ & -0.06 & UWES5 & $\mathbf{0 . 8 2}$ & 0.09 \\
\hline UWES6 & -0.13 & $\mathbf{0 . 7 4}$ & UWES6 & -0.28 & $\mathbf{0 . 7 3}$ \\
\hline UWES7 & $\mathbf{0 . 8 7}$ & -0.02 & UWES7 & $\mathbf{0 . 5 5}$ & 0.43 \\
\hline UWES8 & $\mathbf{0 . 7 4}$ & 0.02 & UWES8 & $\mathbf{0 . 8 1}$ & -0.08 \\
\hline UWES11 & 0.24 & $\mathbf{0 . 5 5}$ & UWES11 & 0.11 & $\mathbf{0 . 7 1}$ \\
\hline UWES12 & 0.26 & $\mathbf{0 . 5 0}$ & UWES12 & 0.22 & $\mathbf{0 . 3 0}$ \\
\hline UWES13 & $\mathbf{0 . 4 5}$ & 0.35 & UWES13 & $\mathbf{0 . 3 8}$ & 0.56 \\
\hline UWES15 & -0.08 & $\mathbf{0 . 5 5}$ & UWES15 & 0.05 & $\mathbf{0 . 6 3}$ \\
\hline
\end{tabular}

The two factors were labelled as follows: a) Factor 1: Vigour/Dedication, and b) Factor 2: Absorption. A target rotation was subsequently carried out on the two pattern matrixes, which resulted in Tucker's phi coefficients of 0.97 (Vigour/Dedication) and 0.92 (Absorption). These coefficients can be regarded as acceptable. Although the construct equivalence of the UWES is acceptable for whites and blacks, the following items were complex: a) Item 3 - "Time flies when I'm working.". This item showed equal loadings on both factors in the white and black groups. Furthermore, the loading could at best be described as moderate. b) Item 13 - "To me, my work is challenging." This item also loaded on both factors in the white and black groups.

The descriptive statistics, alpha coefficients and inter-item correlations of the three factors of the UWES are given in Table 5.

\section{Table 5}

Descriptive statistics and Alpha coefficients of the UWES

\begin{tabular}{|lllllll|}
\hline Item & Mean & $S D$ & Skewness & Kurtosis & $\alpha$ \\
\hline $\begin{array}{l}\text { Vigour/ } \\
\text { dedication }\end{array}$ & 29.09 & 8.93 & -0.75 & 0.23 & 0.87 \\
\hline Absorption & 18.69 & 5.56 & -0.30 & -0.05 & 0.61 \\
\hline
\end{tabular}

From the results in Table 5, it is evident that the scores on the two scales are normally distributed. The internal consistency of Vigour/Dedication is highly acceptable compared to the guideline of 0.70 (Nunnally \& Bernstein, 1994). However, the internal consistency of Absorption is below the guideline of 0.70 . However, Schaufeli, Martinez, et al. (2002) in their cross-national study of students' engagement also reported alpha coefficients for Absorption below the 0.70 range (between 0.65 and 0.73 ).

These findings provide partial support for Hypothesis 1, while Hypothesis 2 is accepted.

\section{5}

\section{Discussion}

The psychometric properties of the UWES were investigated in this study, specifically for emergency medical technicians in the Gauteng Province of South Africa. The objectives were to determine the construct validity and internal 
consistency of the UWES and to test for construct equivalence and bias for the different language groups in the sample of emergency medical technicians.

No evidence for either uniform or nonuniform bias was found for the items of the UWES. This findings confirms the findings of Storm and Rothmann (2003), who found no uniform or non-uniform bias in a South African Police Service sample.

Two factors were extracted by using exploratory factor analysis, namely Vigour/ Dedication and Absorption. However, exploratory factor analysis with target rotations showed that the construct equivalence of the scales was not acceptable. Inspection of the factor loadings revealed that three items $(9,10$ and 14) were problematic. Item 9 ("I feel happy when I am engrossed in my work") is supposed to measure absorption. It is possible that the participants did not understand the word "engrossed". Item 10 ("I am proud of the work that I do") loaded on different factors for whites and blacks. In the white group, it loaded on Vigour/Dedication, where it is supposed to load but in the black group it loaded on Absorption. Item 14 ("I get carried away by my work."), which is supposed to measure Absorption, was also complex, especially in the white group. This item contains a metaphor, which might have been interpreted differently by participants. Therefore, simplifying these items might be necessary in future studies. Item 15 ("I am very resilient, mentally, in my job") loaded on Absorption (instead of Vigour/Dedication where it is supposed to load) in both groups. The word "resilient" might have been misunderstood.

After items 9, 10 and 14 were removed from the analyses, target rotation resulted acceptable Tucker's phi coefficients, which indicate acceptable construct equivalence of the two factors for the white and black emergency medical technicians. However, item 3 ("Time flies when I'm working") seem to be complex. This might have been caused by the fact that a metaphor is used in the item. At least some participants might have found the wording confusing. Item 13 ("To me, my work is challenging") also showed significant cross- loadings on both factors. In the white group, this item loaded higher on Vigour/Dedication, while in the black group it loaded higher on Absorption.

The results of this study suggest that the formulation of some of the items of the UWES are less than optimal. Specific problems encountered with the items include that metaphors are used and that some English words might be difficult to understand. According to Van de Vijver and Leung (1997), metaphors should be avoided in questionnaires. In South Africa, where there are a number of languages and English is a second language for most people, the use of metaphors and the use of uncommon words such as "resilience", "immersed" and "engrossed" in items could have contributed to misunderstandings. Furthermore, while it is often argued that questionnaires should be administered in English because it is regarded as the "business language", the best strategy to improve the construct validity and construct equivalence of the UWES might be to translate it to the official languages of South Africa.

Although studies in Spain and the Netherlands (e.g. Schaufeli, Martinez et al., 2002; Schaufeli, Salanova et al., 2002) confirmed a three-factor structure of work engagement, the results of this study point to a two-factor structure, namely Vigour/ Dedication and Absorption. The internal consistency of the Absorption scale was also not acceptable. Therefore, the question arises whether Absorption should be included in the conceptualisation and measurement of work engagement. Work engagement is primarily characterised by vigour (high energy at work) and dedication (strong identification with work). It is possible that absorption plays a less central role in the work engagement concept (Schaufeli, 2004).

Based on the results of this study, the question arises whether it will be possible to integrate work engagement (i.e. vigour and dedication) and burnout (i.e. exhaustion and cynicism) in one model of work wellness. Exhaustion (low energy) and mental distancing (poor identification) are the hallmarks of burnout that are assessed by the MBI (see Naudé \& 
Rothmann, in press). Vigour (high energy) and dedication (strong identification) as measured by the UWES seem to be opposites of exhaustion and mental distancing (as measured by the MBI).

A limitation of this study is the size of the sample, specifically the distribution of language groups and the sampling method. Future studies could benefit greatly by utilising a random, stratified sample with the proportionate inclusion of all language groups in the sample. Furthermore, future studies should focus on bias and equivalence of the UWES for different language groups.

\section{6}

\section{Recommendations}

According to the results obtained in this study, the use of the UWES is recommended to assess engagement of emergency medical technicians in Gauteng. Although the Absorption scale should not be used before the internal consistency and problems surrounding the construct have not been clarified, the Vigour and Dedication scales could be used to provide data on the work wellness of emergency medical technicians.

It is suggested that future research could focus on the UWES in the emergency services in other provinces in South Africa, to verify the current findings in terms of equivalence. It is also recommended that larger samples with a more powerful sampling method be utilised to enable generalisation of the findings to other similar groups. In future studies some of the items of the UWES (e.g. 3, 9, 10, 14 and 15) could be changed so that it would be more understandable to employees. The UWES should also be translated to other South African languages.

\section{Endnote}

This material is based upon work supported by the National Research Foundation under grant number 2053344.

\section{References}

1 BARNARD, C.P. (1994) "Resilience: A shift in our perception?" American Journal of Family Therapy, 22: 135-44.

2 DIENER, E., SUH, E.M., LUCAS, R.E. \& SMITH, H.I. (1999). "Subjective well-being: Three decades of progress", Psychological Bulletin, 125: 267-302.

3 MASLACH, C. (1993) "Burnout: A multidimensional perspective", In W.B. Schaufeli, C. Maslach \& T. Marek (eds.) Professional Burnout (pp. 19-32) Taylor \& Francis: Washington.

4 MASLACH, C. \& LEITER, M.P. (1997) The Truth about Burnout, Van Nostrand: San Francisco.

5 MASLACH, C., SCHAUFELI, W.B. \& LEITER, M.P. (2001) "Job burnout", Annual Review of Psychology, 52: 397-422.

6 MELLENBERGH, G.J. (1982) "Contingency table models for assessing item bias", Journal of Educational Statistics, 7: 105-118.

7 NUNNALLY, J.C. \& BERNSTEIN, I.H. (1994) "Psychometric Theory" ( ${ }^{\text {rd }}$ ed.) McGraw-Hill: New York.

8 NAUDÉ, J.L.P. \& ROTHMANN, S. (in press). "The validation of the Maslach Burnout Inventory - Human Services Survey for emergency health technicians in Gauteng." South African Journal of Industrial Psychology.

9 POORTINGA, Y.H. (1989) "Equivalence of cross-cultural data: An overview of basic issues", International Journal of Psychology, 24: 737-56.

10 SCHAUFELI, W.B. (2004) "From burnout to engagement", Paper presented at the $2^{\text {nd }}$ South African Work Wellness Conference, March, Potchefstroom.

11 SCHAUFELI, W.B. \& BAKKER, A.B. (2001) "Werk en welbevinden: naar een positieve benadering in de arbeids- en gezondheidspsychologie", [Work and Well-being: Towards a Positive Occupational Health Psychology]. Gedrag en Organizatie, 14: 229-53.

12 SCHAUFELI, W.B., MARTINEZ, I., PINTO, A.M., SALANOVA, M. \& BAKKER, A.B. (2002) "Burnout and engagement in university students: A cross national study", Journal of Cross-Cultural Psychology, 33: 464-481.

13 SCHAUFELI, W.B., SALANOVA, M., GONZÁLEZ-ROMÁ, V. \& BAKKER, A.B. (2002) "The measurement of engagement and burnout: A confirmative analytic approach", Journal of Happiness Studies, 3: 71-92. 
14 SCHAUFELI, W.B., TARIS, T.W., LE BLANC, P.M., PEETERS, M., BAKKER, A.B. \& DE JONGE, J. (2001) "Maakt arbeit gezond?: Op zoek naar de bevlogen werknemer" [Does work make healthy? in search of the engaged worker]. De Psycholoog, 36: 422-28.

15 SELIGMAN, M. (2002) "Positive psychology at work", In C.R. Snyder \& S.J. Lopez (eds.) Handbook of Positive Psychology (pp. 715-28) Oxford University Press: Oxford.

16 SELIGMAN, M.E.P. \& CSIKSZENTMIHALYI, M. (2000) "Positive psychology: An introduction”, American Psychologist, 55(1): 5-14.

17 SNYDER, C.R. \& LOPEZ, S.J. (2002) "Handbook of Positive Psychology", Oxford University Press: Oxford.
18 SPSS Inc. (2003) SPSS 12.0 for Windows, Author: Chicago, IL.

19 STORM, K. \& ROTHMANN, S. (2003). "The validation of the Utrecht Work Engagement Scale in the South African Police Services", South African Journal of Industrial Psychology, 29(4): 62-72.

20 STRÜMPFER, D.J.W. (2001) "Psychofortology: Review of a New Paradigm Marching on", Available: http://www.general.rau.ac.za/psych.

21 VAN DE VIJVER, F. \& LEUNG, K. (1997) Methods and Data Analysis for Cross-Cultural Research, Sage: Thousand Oaks, CA. 